\title{
Expression of MicroRNA in Host Cells Infected with Helicobacter pylori
}

\author{
Jun-Won Chung ${ }^{1}$, Seok Hoo Jeong ${ }^{2}$, Sun Mi Lee ${ }^{3}$, Jhang Ho Pak ${ }^{3}$, Gin Hyug Lee ${ }^{4}$, Jin-Yong Jeong ${ }^{3}$, and Jin-Ho Kim ${ }^{4}$ \\ ${ }^{1}$ Department of Internal Medicine, Gachon University Gil Medical Center, ${ }^{2}$ Department of Internal Medicine, Catholic Kwandong University \\ International St. Mary's Hospital, Incheon, ${ }^{3}$ Department of Convergence Medicine and Asan Institute for Life Sciences, and ${ }^{4}$ Department of \\ Gastroenterology, Asan Medical Center, University of Ulsan College of Medicine, Seoul, Korea
}

Background/Aims: MicroRNAs (miRNAs) regulate gene expression. We assess miRNA regulation by Helicobacter pylori infection and elucidate their role in $\mathrm{H}$. pylori-infected gastric epithelial cells. Methods: The relationship between miRNA expression and DNA methylation was examined. Cells were treated with the nuclear factor-kappaB (NF-kB) inhibitor Bay 11-7082 to determine the relationship between miRNA expression and NF-KB signal transduction. Results: In the negative control cells infected with $H$. pylori 26695 , the expression of six miRNAs was increased, whereas the expression of five miRNAs was decreased. The expression of upregulated miRNAs was increased when the host cells were treated with H. pylori and an NF-KB inhibitor. miR-127-5p, -155, and -181 were associated with increased interleukin 6 (IL-6) secretion in $H$. pylori infected cells treated with anti-miRNA. The expression of miR-155, -127-5p, -195, -216, -206, and -488 increased by approximately 3 -fold following treatment with the methylation inhibitor Aza. Conclusions: We found novel miRNAs in H. pylori-infected negative control cells using miRNA microarrays. Upregulated miRNA expression was inversely related to the transcription of NF-KB. miR-195 and miR-488 appear to play a pivotal role in controlling IL-6 activity in $H$. pylori infection. miRNA expression in $H$. pylori infection was affected by methylation. (Gut Liver 2017;11:392-400)

Key Words: Helicobacter pylori; MicroRNAs; NF-kappa B; Cytokines; Negative control cell lines

\section{INTRODUCTION}

Helicobacter pylori is a Gram-negative helical bacterium that causes chronic active gastritis and peptic ulcers and is a major risk factor for gastric cancer, as demonstrated in several animal and epidemiological studies. ${ }^{1} \mathrm{H}$. pylori is classified as a definite (type I) carcinogen because of its prevalence in 80\% of gastric cancers according to International Agency for Research on Cancer of the World Health Organization. However, the relationship of molecular mechanism between $H$. pylori infection and gastric cancer is not understood comprehensively.

Over half of the world's population is infected with $H$. pylori. The infection rate is relatively high in South Korea, where 50\% of 5-year-olds are already infected, and 70\% to $80 \%$ of those aged 10 to 20 years are carriers. ${ }^{2}$ Consequently, South Koreans have a high frequency of gastric diseases such as gastric cancer compared with Western populations. Primary H. pylori infection occurs predominantly during adolescence and, if not eradicated, will most likely remain as a permanent lifetime infection. Moreover, there is $20 \%$ variation in the $H$. pylori genome in different host species. ${ }^{3}$ H. pylori infection in humans appears to cause inflammation in the gastric mucosa for decades, and the species adapts to the host environment through genetic recombination. ${ }^{4,5}$ The chronic inflammation can lead to precancerous lesions via atrophic gastritis and intestinal metaplasia; consequently, $H$. pylori plays an important role in the preliminary stages of gastric cancer. ${ }^{6,7}$ However, the fact that gastric cancer occurs in only a proportion of the population infected with $H$. pylori suggests that the disease involves many stages occurring over a long period of time and intricate relationships involving

Correspondence to: Jin-Yong Jeong ${ }^{\mathrm{a}}$ and Jin-Ho Kim ${ }^{\mathrm{b}}$

${ }^{a}$ Department of Convergence Medicine and Asan Institute for Life Sciences, Asan Medical Center, University of Ulsan College of Medicine, 88 Olympic-ro 43-gil, Songpa-gu, Seoul 05505, Korea

Tel: +82-2-3010-4105, Fax: +82-2-3010-4182, E-mail: jyjeong@amc.seoul.kr

${ }^{b}$ Department of Gastroenterology, Asan Medical Center, University of Ulsan College of Medicine, 88 Olympic-ro 43-gil, Songpa-gu, Seoul 05505, Korea

Tel: +82-2-3010-3185, Fax: +82-2-476-0824, E-mail: jkim@amc.seoul.kr

Received on May 24, 2016. Revised on August 17, 2016. Accepted on September 5, 2016. Published online February 17, 2017

pISSN 1976-2283 eISSN 2005-1212 https://doi.org/10.5009/gnl16265

Jun-Won Chung and Seok Hoo Jeong contributed equally to this work as first authors.

(a) This is an Open Access article distributed under the terms of the Creative Commons Attribution Non-Commercial License (http://creativecommons.org/licenses/by-nc/4.0) which permits unrestricted non-commercial use, distribution, and reproduction in any medium, provided the original work is properly cited. 
the virulence of $H$. pylori, the susceptibility and diet of the host, and environmental factors. Despite much research, the mechanism of the involvement of $H$. pylori in gastric cancer has not yet been determined fully.

A microRNA (miRNA) is a small noncoding RNA composed of 19 to 22 nucleotides that binds to the 3' untranslated regions of specific target messenger RNAs (mRNAs), suppressing their translation and promoting their degradation. ${ }^{8}$ This is how miRNAs regulate over 30\% of all genes and play important roles in cell proliferation, cell death, stress resistance, fat metabolism, development, differentiation, and so forth. ${ }^{9}$ Several recent studies have shown that miRNAs participate in human tumorigenesis as tumor suppressors or oncogenes. ${ }^{10-13}$ In molecular mechanism of gastric cancer, novel result has often been confused due to genetic and pathologic heterogeneity of clinical sample. In this regard, animal studies of gastric cancer serve dependable alternatives. A recent study of mice infected with $H$. pylori found significant thickening of the stomach muscle layers and a marked decline in the expression of the muscle-specific miRNAs miR-1 and miR-133. ${ }^{14}$ Another study demonstrated associations of $H$. pylori with several diseases, such as duodenal ulcers. ${ }^{15}$

Although the effects of $H$. pylori infection on human gastric diseases are well known, less is known about the effects of $H$. pylori infection on miRNA expression. ${ }^{16-18}$ Therefore, this study analyzed miRNA expression in $H$. pylori-infected host cells and how miRNAs affect the pathogenicity of $H$. pylori.

\section{MATERIALS AND METHODS}

\section{Cell and $H$. pylori cultures}

The negative control cells were incubated in RPMI 1640 medium (Invitrogen, Carlsbad, CA, USA) supplemented with 10\% fetal bovine serum and $100 \mathrm{U} / \mathrm{mL}$ penicillin in a humidified incubator containing $5 \% \mathrm{CO}_{2}$ at $37^{\circ} \mathrm{C}$. H. pylori strains 26695 (cagA+, vacA s1a/m1), 43504 (cagA+, vacA s1b/m1), J99, and SS1 (cagA+, vacA s2/m2) were incubated in Brucella broth agar with $5 \%$ sheep's blood. $H$. pylori was added to the negative control cells at 100 bacteria cells/negative control cell. The negative control cells were collected 6 and 24 hours after $H$. pylori infection.

\section{Bay 11-7082 treatment}

To determine the relationship between miRNA expression and nuclear factor-kappaB $(\mathrm{NF}-\kappa \mathrm{B})$ signal transduction, the negative control cells were treated with $10 \mu \mathrm{M}$ Bay 11-7082, a NF- $\kappa \mathrm{B}$ inhibitor or medium control for 1 hour. Cells were washed and either untreated of treated with $H$. pylori 26695 for 6 hours or with $50 \mathrm{ng} / \mathrm{mL}$ interleukin 6 (IL-6) cytokine for 8 hours. miRNA expression was quantified using real-time polymerase chain reaction (RT-PCR).

\section{3. miRNA microarrays}

RNA was extracted using TRIzol (Invitrogen) and analyzed quantitatively and qualitatively using the NanoDrop ND-1000 spectrophotometer (NanoDrop Technologies, Wilmington, DE, USA) and Agilent 2100 Bioanalyzer (Agilent Technologies, Santa Clara, CA, USA). miRNA microarray were performed using the PANArray ${ }^{\mathrm{TM}}$ miRNA expression profiling kit at Panagene Co., Ltd. (Daejeon, Korea). The microarray contained positive control probes and 135 probes for cancer-related human miRNAs and generated fluorescent miRNAs with a sample input of 400 ng of total RNA (control cell and H. pylori infected cell).

\section{5'-Aza-2'-deoxycytidine (5-aza-dC) treatment}

To examine the role of methylation in the regulation of miRNA expression, the negative control cells were cultured with $2.5 \mu \mathrm{M}$ 5-Aza-dC for 4 days, with $0.25 \mu \mathrm{M}$ trichostatin A (TSA) only for 1day or for 4 days with $2.5 \mu \mathrm{M}$ 5-Aza-dC, to which was added $0.25 \mu \mathrm{M}$ TSA after day 3. miRNA expression in the negative control cells was quantified using RT-PCR. 5-Aza- $\mathrm{dC}^{19}$ and TSA $^{20}$ were purchased from Sigma (St. Louis, MO, USA).

\section{Cell transfection}

Transfection was performed using Lipofectamine 2000 (Invitrogen). The negative control cells were transfected with $50 \mathrm{nM}$ of the miRNA inhibitors or $10 \mathrm{nM}$ of the analogues, along with the respective control groups for 48 hours. $H$. pylori infection was continued for 24 hours. IL-6 levels in culture media were measured by enzyme-linked immunosorbent assay (ELISA).

\section{RNA extraction and quantitative RT-PCR}

Total RNA from the negative control cells was isolated using a miRNeasy mini kit (Qiagen, Mississauga, ON, Canada), as described in the product manual, and quantified using the NanoDrop 1000 spectrophotometer (NanoDrop Technologies). The total RNA $(1 \mu \mathrm{g})$ was subjected to a stem-loop reverse

Table 1. Sequence of Oligonucleotides Used in This Study

\begin{tabular}{ll}
\hline \multicolumn{1}{c}{ Name } & \multicolumn{1}{c}{ Sequences } \\
\hline hsa-miR-17-3p & 5' ACUGCAGUGAAGGCACUUGUAG \\
hsa-miR-34a & 5' CAAUCAGCAAGUAUACUGCCCU \\
hsa-miR-103 & 5' AGCAGCAUUGUACAGGGCUAUGA \\
has-miR-127-5p & 5' CUGAAGCUCAGAGGGCUCUGAU \\
hsa-miR-141 & 5' CAUCUUCCAGUACAGUGUUGGA \\
hsa-miR-195 & 5' CCAAUAUUGGCUGUGCUGCUCC \\
hsa-miR-196a & 5' UAGGUAGUUUCAUGUUGUUGGG \\
hsa-miR-206 & 5' UGGAAUGUAAGGAAGUGUGUGG \\
hsa-miR-216 & 5' UAAUCUCAGCUGGCAACUGUGA \\
hsa-miR-488 & 5' CCCAGAUAAUGGCACUCUCAA \\
hsa-Let-7i & 5' UGAGGUAGUAGUUUGUGCUGUU \\
\hline
\end{tabular}


transcription reaction using miScript primer assay (Table 1). To detect the expression of mature miRNAs, quantitative RT-PCR (qRT-PCR) were performed using the Qiagen miScript PCR System according to the manufacturer's instructions. The reaction was performed under the following condition: initial incubation step of 15 minutes at $95^{\circ} \mathrm{C}$, followed by 40 cycles of $94^{\circ} \mathrm{C}$ for 15 seconds, $55^{\circ} \mathrm{C} 30$ seconds, $70^{\circ} \mathrm{C}$ for 30 seconds. The miRNA expression in both $H$. pylori-infected and uninfected cells was measured using the LightCycler 480 (Roche Diagnostics, Seoul, Korea). RNU6B was used as an internal comparison for data normalization, and the difference in the expression of miRNA was calculated using cycle threshold values.

\section{Enzyme-linked immunosorbent assay}

To measure cytokine release, IL-6 levels (R\&D Systems, Minneapolis, MN, USA) in the collected supernatant were measured using the DuoSet ELISA Development System (R\&D Systems).

\section{RESULTS}

\section{1. miRNA expression in response to $H$. pylori infection}

Six miRNAs (miR-196a, -127-5p, -206, -216, -488, and -195) showed increased expression, and five miRNAs (miR-34a, -141, $-17-3 p, 103$, and let-7i) showed decreased expression in the negative control cells infected with $H$. pylori 26695. The increased miRNA expression seen with $H$. pylori 26695 was also apparent with the 43504, J99, and SS1 H. pylori strains which were seen similar expression patterns, although SS1 resulted in relatively lower expression levels (Fig. 1A). Similarly, the decreased miRNA expression seen with $26695 \mathrm{H}$. pylori was also seen with the other strains, although the amplitude of the decrease induced by strain 43504 was low (Fig. 1B).

\section{The relationship between miRNA expression and NF-kB signaling}

To identify the relationship of the NF- $\mathrm{B}$ pathway with the resulting miRNA expression following $H$. pylori infection, we pretreated the negative control cells with a NF- $\kappa B$ inhibitor (BAY-117082) before exposing them to $H$. pylori. As shown in Fig. 2A, treatment with BAY-117082 alone did not change the miRNA expression, while the expression of these miRNAs was significantly increased following $H$. pylori infection.

Following $H$. pylori infection, the expression levels of miR$34 a,-141,-17-3 p$, and Let-7i were decreased or relative little change. There was no change in miRNA expression following treatment with BAY-117082 only (Fig. 2B).

\section{The secretion of inflammatory cytokine due to specific miRNAs expressed in $H$. pylori infection}

We investigated whether the secretion of inflammatory cytokine was due to specific miRNAs expressed following $H$. pylori infection (Fig. 3). In this experiment, the negative control cells were transfected with miRNA analogues or inhibitors for 24 hours before $H$. pylori infection, and the quantity of IL-6 secreted within the cells was measured. The secretion of IL- 6 was increased comparing with the medium when cells were treated with anti-miRNAs targeting miR-127-5p, -155, and -181, which were the miRNAs that showed increased expression with H. pylori infection. In comparison, anti-miRNA miR-195 and miR-488 did not alter IL-6 secretion (anti-miR-195 and -488)
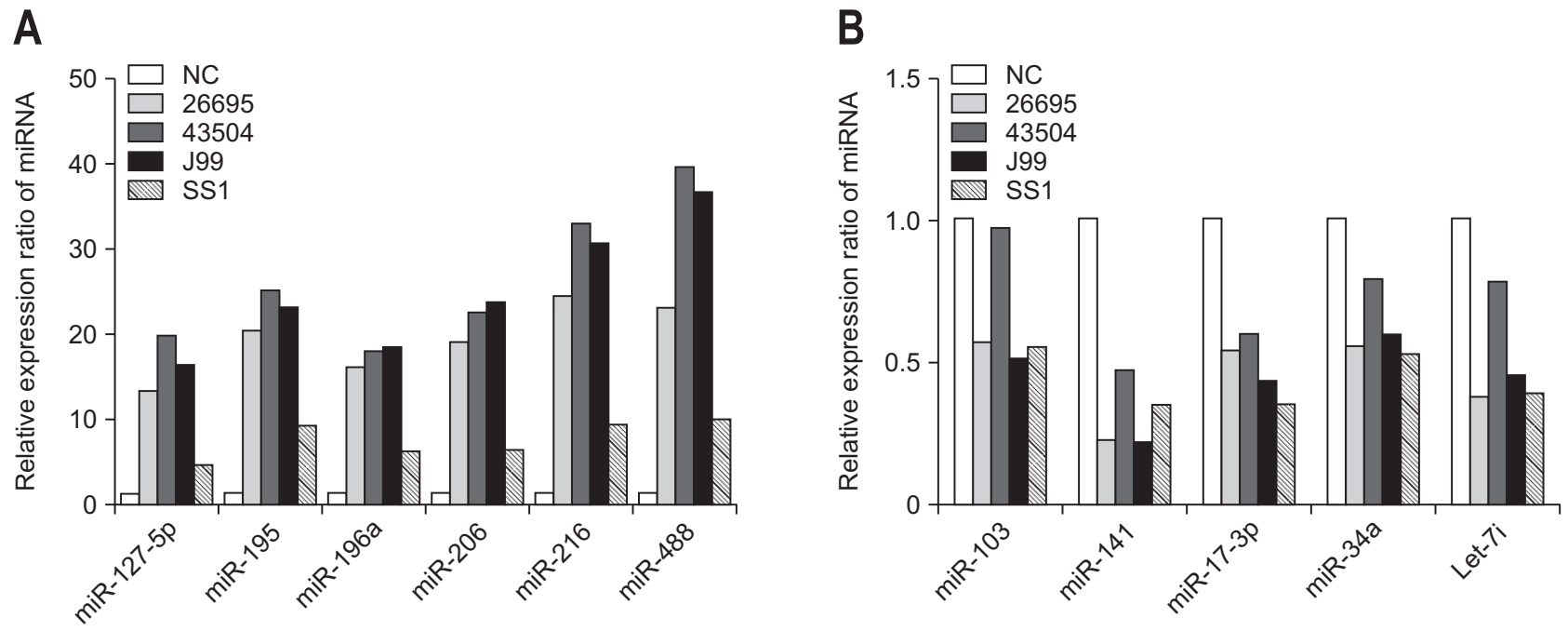

Fig. 1. MicroRNA (miRNA) expression in response to Helicobacter pylori infection. miRNA expression was assayed by quantitative real-time polymerase chain reaction (qRT-PCR). (A) The increased miRNA expression observed with H. pylori 26695 was also apparent in the 43504, J99, and SS1 H. pylori strains; however, SS1 resulted in relatively lower expression levels. (B) The decreased miRNA expression observed with $26695 \mathrm{H}$. pylori was also observed with the other strains; however, the amplitude of the decrease induced by strain 43504 was low.

$\mathrm{NC}$, negative control. 

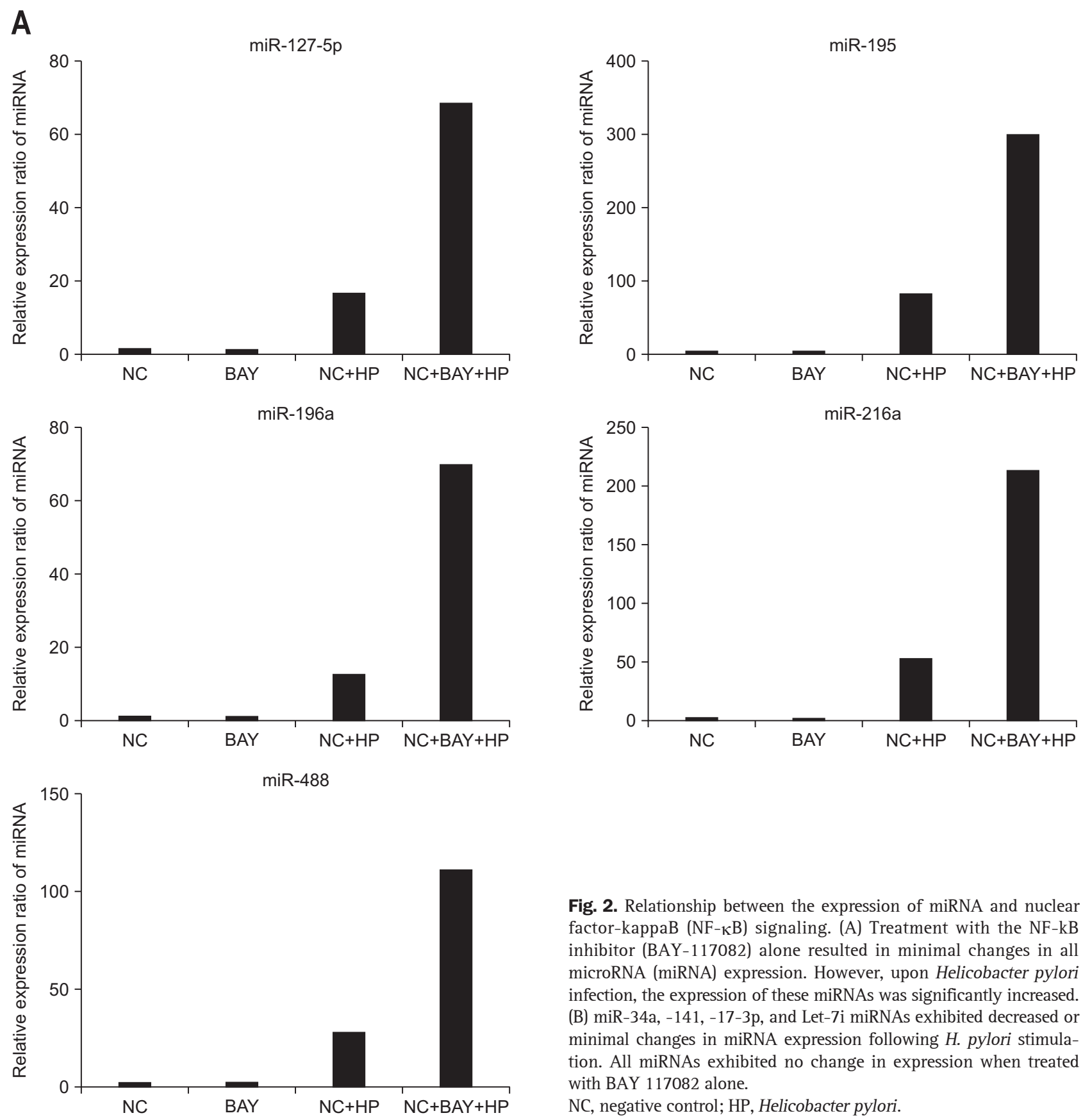

Fig. 2. Relationship between the expression of miRNA and nuclear factor-kappaB (NF- $\kappa B$ ) signaling. (A) Treatment with the NF-kB inhibitor (BAY-117082) alone resulted in minimal changes in all microRNA (miRNA) expression. However, upon Helicobacter pylori infection, the expression of these miRNAs was significantly increased. (B) miR-34a, -141, -17-3p, and Let-7i miRNAs exhibited decreased or minimal changes in miRNA expression following $H$. pylori stimulation. All miRNAs exhibited no change in expression when treated with BAY 117082 alone.

NC, negative control; HP, Helicobacter pylori.

comparing with the medium upon H. pylori infection (Fig. 3A). Similarly, with anti-miRNAs targeting miR-34a and Let-7i, both of which showed decreased expression with $H$. pylori infection, the secretion of IL- 6 was decreased by $H$. pylori infection comparing with the medium (Fig. 3B).

\section{Association of miRNAs with epigenetic modifications in host cells infected with $H$. pylori}

The miRNAs miR-155, -127-5p, -195, -216, -206, and -488 all showed increased expression with $H$. pylori infection, and their expression was increased 3-fold after cells were treated with the methylation inhibitor Aza. In comparison, there was no

change in expression in cells treated with TSA (Fig. 4A).

The miRNAs that showed decreased expression following $H$. pylori infection showed different results compared with the miRNAs that showed increased expression (Fig. 4B). miR-34a appears to be affected by methylation, because its expression increased by over 3-fold in conditioned cells. However, miR-34a does not appear to play a role in H. pylori infection, because its expression did not change much by $H$. pylori infection. miR-17$3 p$ and miR-106b were not affected by methylation without $H$. pylori infection, but after $H$. pylori infection, they were clearly affected by methylation. Let-7i showed decreased expression in the negative control cells treated with the methylation inhibi- 

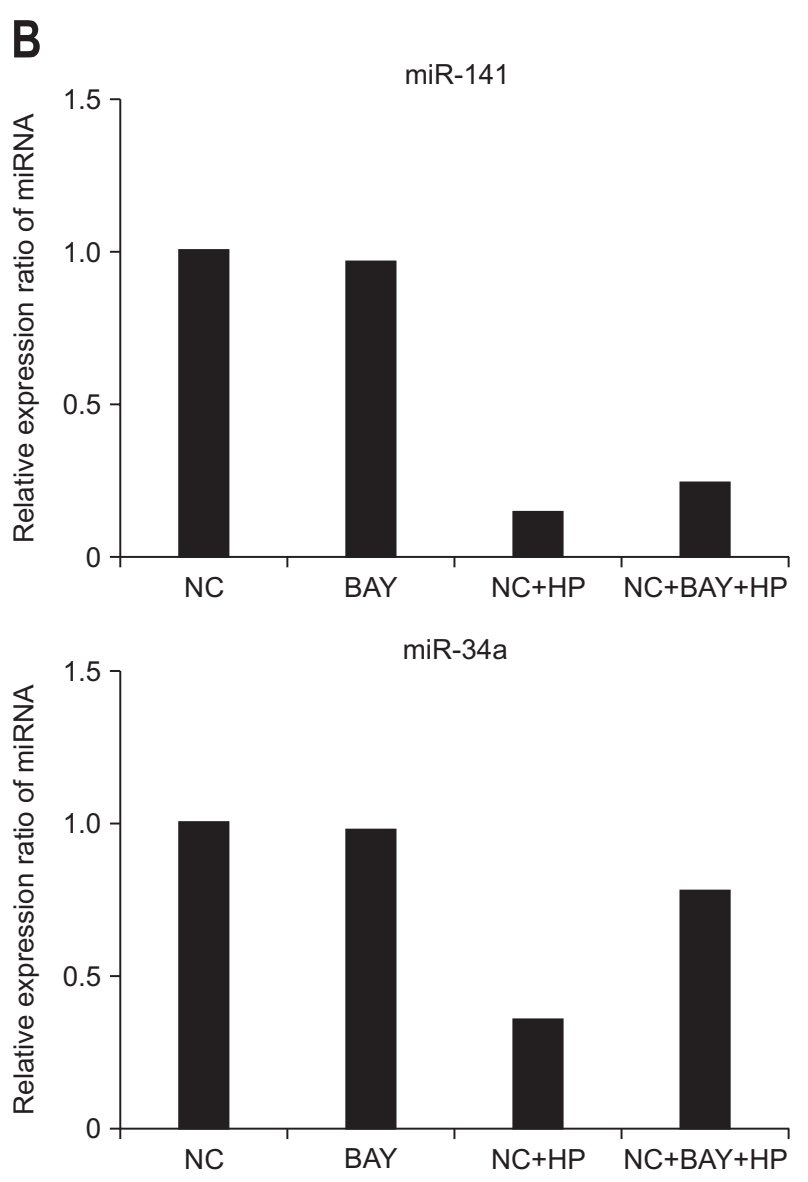

Fig. 2. Continued.

tor (Aza) or acetylation inhibitor (TSA), and following $H$. pylori infection, its expression was decreased.

\section{Association of miRNA expression with $H$. pylori-related proinflammatory cytokine}

H. pylori infection stimulates the secretion of inflammatory cytokine. Therefore, we examined whether $H$. pylori-related proinflammatory cytokine affect miRNA expression (Fig. 5). When the negative control cells were treated with IL-6, H. pylori infection had no significant effect on expression of miRNAs, except miR-488, which increased 2-fold.

\section{DISCUSSION}

In this study, the expression levels of miR-196, -127-5p, $-206,-216,-488,-195$, and -181 were increased by $H$. pylori infection, and miR-196 and miR-488 controlled IL-6 secretion following $H$. pylori infection. Intracellular epigenetic modifications affect the expression of many genes and the miRNAs. ${ }^{21}$ To determine whether $H$. pylori infection affects miRNA expression following epigenetic modifications, we examined changes in miRNA expression in cells conditioned with a methylation inhibitor (Aza) or acetylation inhibitor (TSA) using qRT-PCR.
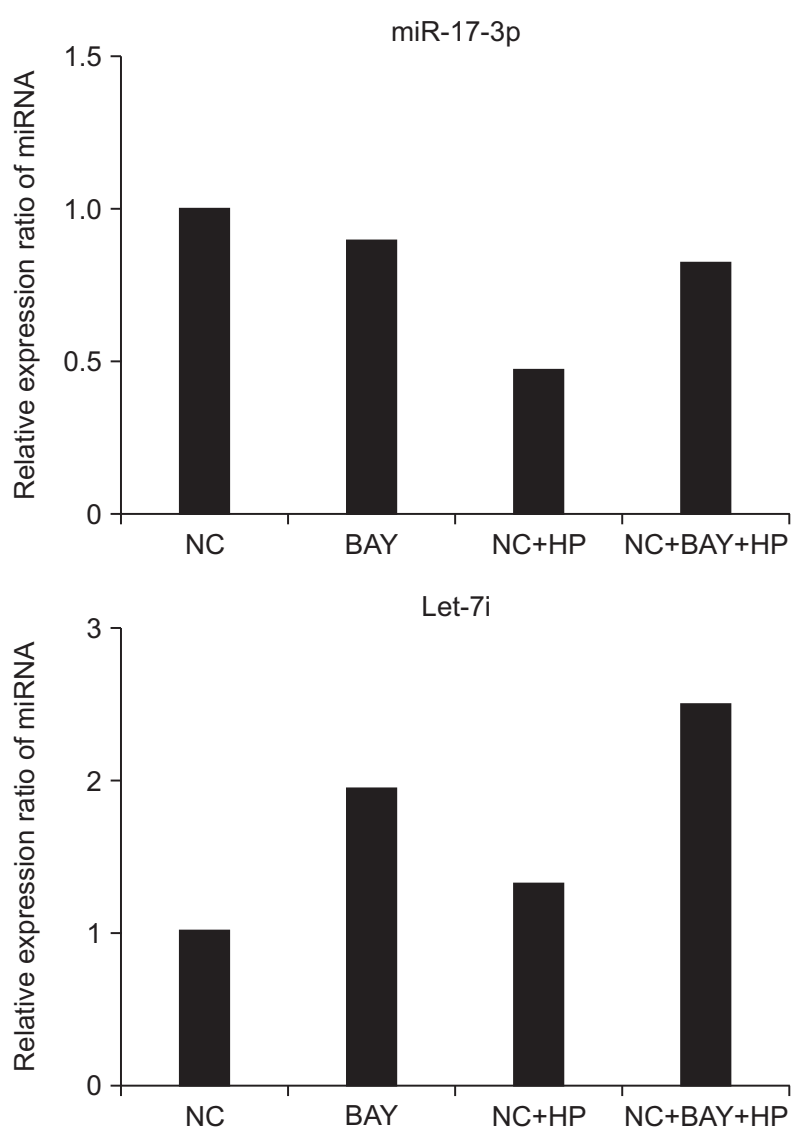

Present study showed miRNA expression was affected by methylation, but not by acetylation, and $H$. pylori infection inhibited the methylation of miRNAs, promoting their expression.

Studies have examined the expression and function of miR155, -196a, 103, and -200c. ${ }^{10,22-24} \mathrm{NF}-\kappa \mathrm{B}$ is a protein complex involved in DNA transcriptional control at the cellular level and is affected by stress, free radicals, and microbial or viral antigens. The expression of miR-155 is affected by NF- $\kappa$ B and $\mathrm{AP}-1$, and it inversely affects the secretion of cytokine such as IL-8 and growth-related oncogene (GRO)- $\alpha$. These results are in accordance with our previous results. Although the NF- $\kappa B$ signal transduction pathway has little effect on the expression of miR196, -127-5p, -206, -216, -488, -195, -181 when not infected by $H$. pylori, following $H$. pylori infection, they are negatively correlated with the $\mathrm{NF}-\kappa \mathrm{B}$ signal transduction pathway. Following $H$. pylori infection, the increase in miR146 a expression is dependent on NF- $\mathrm{BB}$. miR-146 also inhibits the expression of $\mathrm{IL}-1$ receptor-associated kinase 1 and tumor necrosis factor (TNF) receptor-associated factor 6, which are target genes of miR-146. miR-146 has also been reported to play a role in the negative feedback of IL-8, GRO- $\alpha$, and macrophage inflammatory protein 3a during inflammation induced by $H$. pylori. ${ }^{25,26}$ A study of the function of miR-218 showed decreased 
A

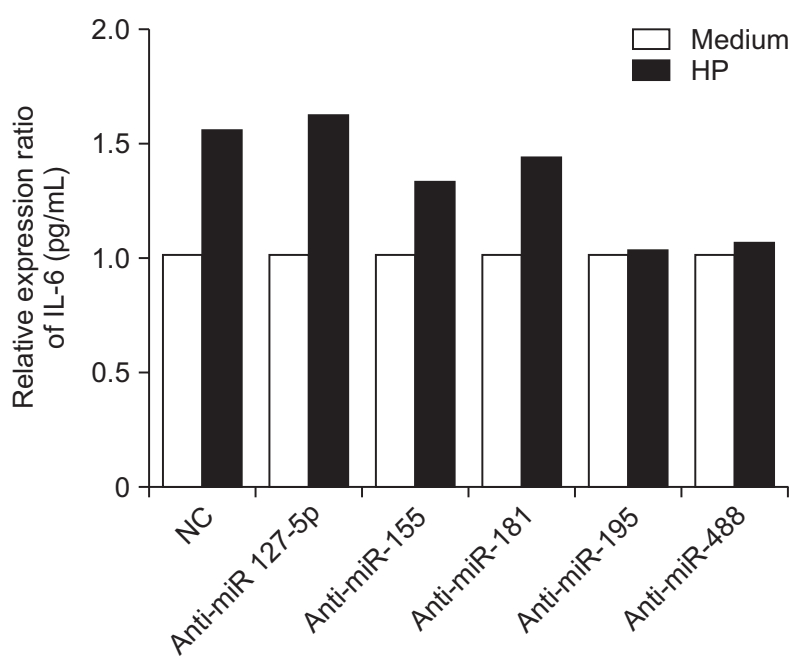

B

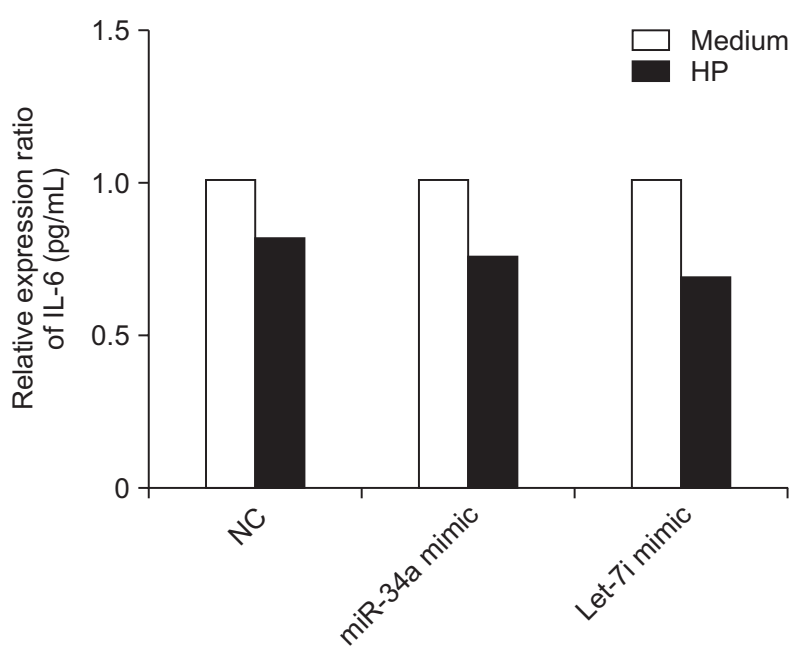

Fig. 3. Secretion of inflammatory cytokines due to specific microRNA (miRNA) expressed upon Helicobacter pylori infection. (A) miR-127-5p, -155 , and -181 , which are miRNAs that exhibited increased expression during $H$. pylori infection, demonstrated increased secretion of interleukin 6 (IL-6) when treated with anti-miRNA and H. pylori infection. However, miR-195 and miR-488 exhibited minimal change in IL-6 secretion despite the application of anti-miRNA inhibitors (anti-miR-195, -488) during $H$. pylori infection. (B) Upon treatment with anti-miRNA inhibitors of miR-34a and Let-7i, both of which exhibited decreased expression during $H$. pylori infection, secretion of IL-6 was decreased during $H$. pylori infection. These results indicate that these miRNAs have no effect on the intracellular secretion of IL-6 during $H$. pylori infection.

$\mathrm{HP}$, Helicobacter pylori; NC, negative control.

A

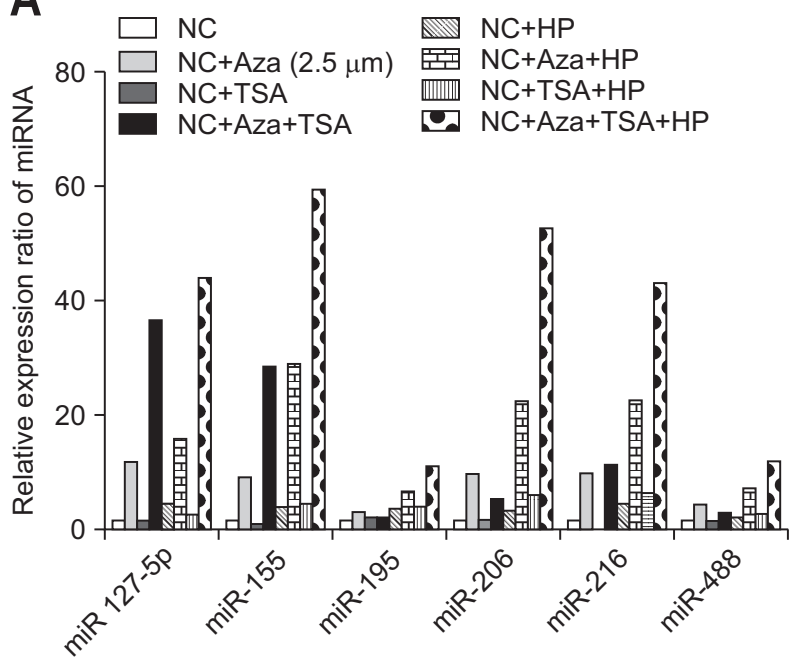

B

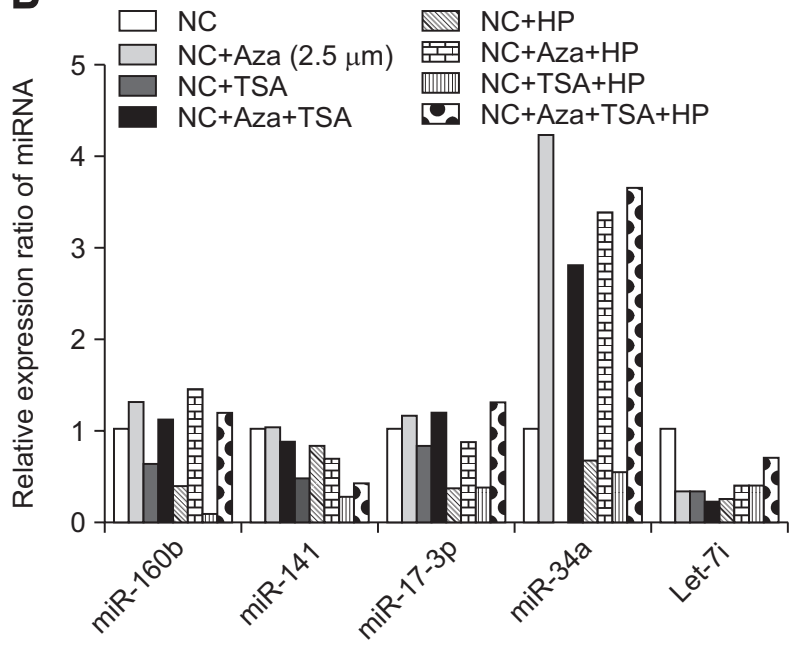

Fig. 4. Association of microRNA (miRNA) and epigenetic modification in host cells infected with Helicobacter pylori. (A) miR-155, -127-5p, -195, -216, -206, and -488, all of which exhibited increased expression during $H$. pylori infection, demonstrated a 3-fold increase in expression upon treatment with the methylation inhibitor Aza. However, trichostatin A (TSA) treatment resulted in minimal changes. (B) miR-34a appeared to be affected by methylation when cells were treated with Aza. miR-34a exhibited a greater than 3-fold increase in expression. However, miR-34a is likely not involved in $H$. pylori infection because it did not exhibit at considerable change in expression upon $H$. pylori infection. miR-17-3p and miR-106b were not affected by methylation when not subject to $H$. pylori infection. However, these miRNAs were distinctly affected by methylation during $H$. pylori infection. In the case of Let-7i, decreased expression was observed when the negative control cell strain was treated with the methylation inhibitor (Aza) and/or acetylation inhibitor (TSA).

NC, negative control; HP, Helicobacter pylori.

expression on mucosa infected by $H$. pylori and in gastric cancer, compared with normal gastric mucosa. H. pylori infection was also reported to decrease the expression of miRNA-218 and increase the transcriptional activity of $\mathrm{NF}-\kappa \mathrm{B}$, which could po- tentially increase the occurrence of gastric cancer. ${ }^{27}$

Overexpression of forkhead box M1 attenuated the expression of miR-370 in gastritis and gastric cancer, which resulted in $H$. pylori-infected gastritis progressing to gastric cancer via 

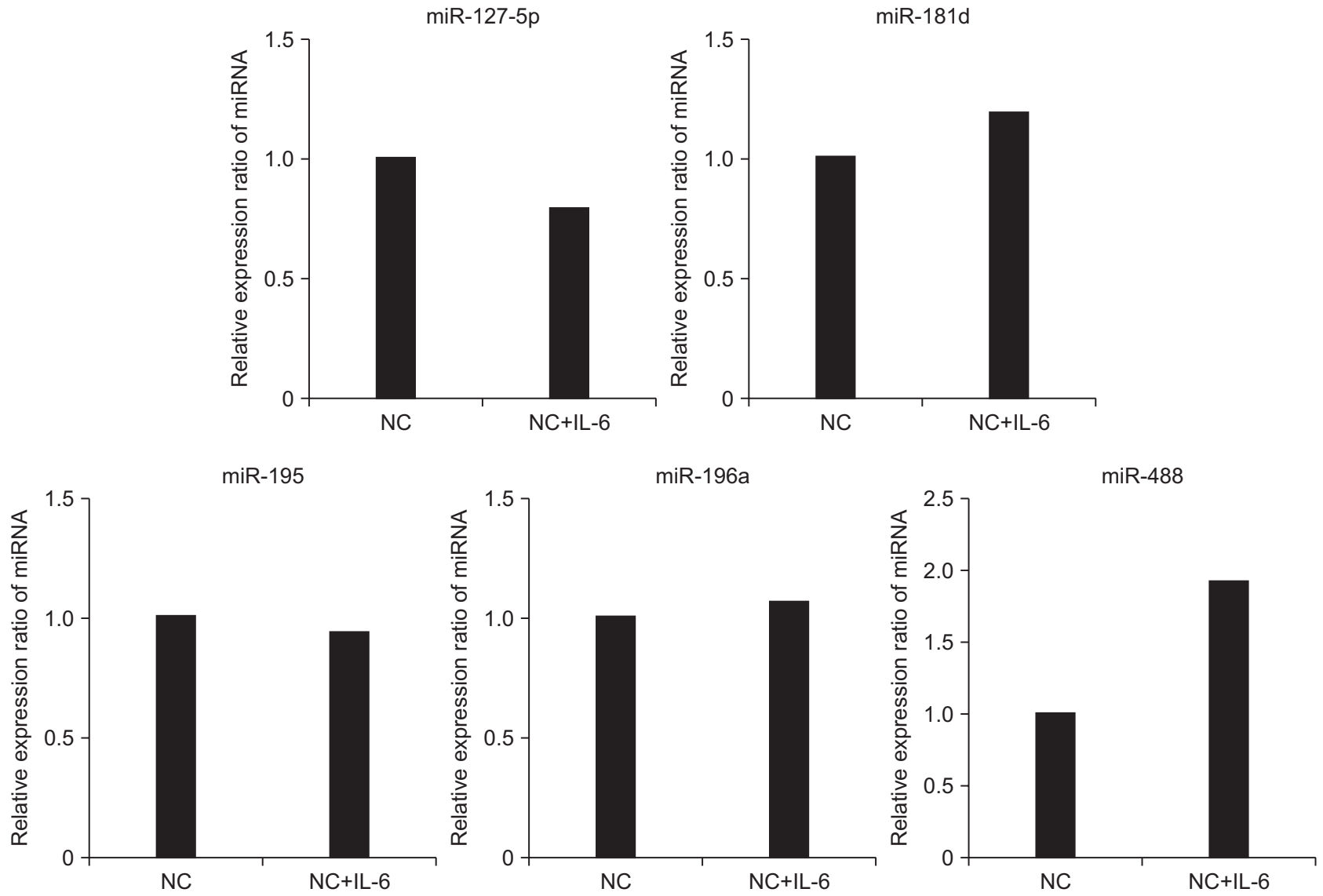

Fig. 5. Association of microRNA (miRNA) expression with Helicobacter pylori-related proinflammatory cytokine. When negative control cells without $H$. pylori infection were treated with interleukin 6 (IL-6), the expression of miRNA was not significantly affected. However, miR-488 exhibited a 2-fold increase.

$\mathrm{NC}$, negative control.

the influence of P27Kip $1 .{ }^{28}$ Ishimoto et al. ${ }^{29}$ demonstrated de novo expression of CD44 variant 9 (CD44 v9) and downregulation of miR-328 in gastric mucosa infected by $H$. pylori adjacent to gastric cancer, compared with uninfected gastric mucosa adjacent to gastric cancer.

The let-7i which is one of the tumor suppressor miRNAs has been identified to be downregulated in prostate cancer, lung cancer, and gastric cancer. Also, the let-7i correlates with tumor progression. ${ }^{30,31}$ In this regard, the losing expression of Let $7 \mathrm{i}$ after $H$. pylori infection would increase the risk of the development of gastric cancer cell.

CagA is an $H$. pylori virulence factor and a type-four secretion system inserted into the host cell. The intracellular CagA undergoes tyrosine phosphorylation via the src family kinases and in turn binds to SHP-2 to activate phosphatase. ${ }^{32,33}$ In gastric epithelial cells, CagA activates $\mathrm{NF}-\kappa \mathrm{B}$ and participates in the secretion of inflammatory cytokine. CagA protein triggers time-dependent induction of the expression of IL-8 caused by H. pylori infection and that Shp-2- and c-Met- are generated nondependently through Ras $\rightarrow$ Raf $\rightarrow$ Mek $\rightarrow$ Erk $\rightarrow$ NF- $\kappa \mathrm{B}$ signaling. ${ }^{34}$
In an experiment using CagA transgenic mice, CagA induced the attenuation of let-7 and upregulation of Ras, which resulted in gastric carcinogenesis. CagA upregulates c-myc, DNA methyltransferase 3B, and enhancer of zeste homologue 2 and downregulates miR-26a and miR-101, leading to reduced expression of let- $7 .^{35}$

When miR-127-5p, -155, and -181 were treated with antimiRNA, the secretion of IL- 6 was increased in the presence of $H$. pylori infection. Since miR-195 and miR-488 did not increase the secretion of IL-6 following $H$. pylori infection, we postulate that they are factors controlling IL-6 secretion. A study comparing biopsies from $H$. pylori-positive and -negative patients reported significant associations among cytokine such as IL-1 $\beta$, IL-6, TNF- $\alpha$, which were increased by $H$. pylori infection, and miR-7b, -200c, -375 , and $-103 .^{36}$ It was also reported that overexpression of miR-155 had a negative feedback effect on the expression of IL-8 and growth-associated tumor genes. ${ }^{23}$

$H$. pylori infection also induces abnormal DNA methylation in the gastric epithelium. In our study, because the effects of methylation were more noticeable with $H$. pylori infection, $H$. pylori infection likely promotes the expression of the miRNAs by in- 
hibiting methylation of their promotors. miR-17-3p and miR106b, which showed decreased expression, may not be affected much by methylation in the absence of $H$. pylori infection; following $H$. pylori infection, however, they were significantly affected by methylation. miR34b/c are novel tumor suppressants frequently inactivated by DNA methylation in gastric tumors; methylation of miR34b/c is associated with epigenetic deficits and predicts the risks of gastric cancer. ${ }^{37}$ Ando et al. ${ }^{38}$ examined the relationship among abnormal DNA methylation accumulation in gastric mucosa appearing normal following $H$. pylori infection and the epigenetic field defect and miRNAs associated with the occurrence of gastric tumors; they reported high methylation in the gastric tumor patients and H. pylori-infected groups. They also reported that methylation-inactivation of miRNA genes contributed to the field defect of gastric cancer. ${ }^{38}$ Another study recently founded that miR-490-3p silenced by aberrant DNA methylation reactivates SMARCD1, a SWI/SNF chromatin remodeling complex subunit; they also reported a link among $H$. pylori, chromatin remodeling, and gastric carcinogenesis. ${ }^{39}$ miR-210 levels influenced by the hypermethylation status of several CpG sites are attenuated by $H$. pylori infection. This activated stathmin 1 and dimethyladenosine transferase 1 , which resulted in the proliferation of gastric epithelial cells, including cancer, in chronic $H$. pylori infection. ${ }^{40}$ In present study, those candidate miRNAs might be increased or decreased confined in only gastric epithelial cells, because the whole experiments with normal gastric cell line could not redo.

In summary, we determined which miRNAs are increased or decreased in expression by $H$. pylori infection and found a negative correlation between the miRNAs that showed increased expression and the NF- $\kappa \mathrm{B}$ signal transduction pathway. Some of the miRNAs (miR-195 and miR-488) controlled the secretion of IL- 6 following $H$. pylori infection. By inhibiting the methylation of miRNA promotors, the expression of certain miRNAs was stimulated.

To establish the function of miRNA in more detail, further experiments should be conducted to examine the relationships between the expression of cytokine IL-8, TNF- $\alpha$, and IL-1 $\beta$ and miRNAs. In the future, we will examine the function and expression of miRNAs discovered in this research in vitro and how they change in both $H$. pylori-infected and noninfected gastric mucosa.

\section{CONFLICTS OF INTEREST}

No potential conflict of interest relevant to this article was reported.

\section{ACKNOWLEDGEMENTS}

This work was supported by a grant (2013-348) from the Asan Institute for Life Sciences, Asan Medical Center, Seoul,
Korea.

\section{REFERENCES}

1. IARC Working Group on the Evaluation of Carcinogenic Risks to Humans. Infection with Helicobacter pylori. In: IARC Working Group on the Evaluation of Carcinogenic Risks to Humans, ed. Schistosomes, liver flukes and Helicobacter pylori. Geneva: World Health Organization, 1994:177-220.

2. Yim JY, Kim N, Choi SH, et al. Seroprevalence of Helicobacter pylori in South Korea. Helicobacter 2007;12:333-340.

3. Israel DA, Salama N, Krishna U, et al. Helicobacter pylori genetic diversity within the gastric niche of a single human host. Proc Natl Acad Sci U S A 2001;98:14625-14630.

4. Nathan C, Ding A. Nonresolving inflammation. Cell 2010;140:871882

5. Khan MK, Bemana M. Association of Helicobacter pylori infection and gastric carcinoma. Mymensingh Med J 2012;21:80-84.

6. Parsonnet J. Helicobacter pylori and gastric cancer. Gastroenterol Clin North Am 1993;22:89-104.

7. Blaser MJ, Atherton JC. Helicobacter pylori persistence: biology and disease. J Clin Invest 2004;113:321-333.

8. Bartel DP. MicroRNAs: target recognition and regulatory functions. Cell 2009;136:215-233.

9. Ambros V. The functions of animal microRNAs. Nature 2004;431:350-355.

10. Okubo M, Tahara T, Shibata T, et al. Association between common genetic variants in pre-microRNAs and gastric cancer risk in Japanese population. Helicobacter 2010;15:524-531.

11. Bou Kheir T, Futoma-Kazmierczak E, Jacobsen A, et al. miR-449 inhibits cell proliferation and is down-regulated in gastric cancer. Mol Cancer 2011;10:29.

12. Craig VJ, Cogliatti SB, Rehrauer H, Wündisch T, Müller A. Epigenetic silencing of microRNA-203 dysregulates ABL1 expression and drives Helicobacter-associated gastric lymphomagenesis. Cancer Res 2011;71:3616-3624.

13. Li N, Tang B, Zhu ED, et al. Increased miR-222 in H. pyloriassociated gastric cancer correlated with tumor progression by promoting cancer cell proliferation and targeting RECK. FEBS Lett 2012;586:722-728.

14. Saito Y, Suzuki H, Tsugawa H, et al. Dysfunctional gastric emptying with down-regulation of muscle-specific microRNAs in Helicobacter pylori-infected mice. Gastroenterology 2011;140:189198.

15. Lario S, Ramírez-Lázaro MJ, Aransay AM, et al. microRNA profiling in duodenal ulcer disease caused by Helicobacter pylori infection in a Western population. Clin Microbiol Infect 2012;18:E273E282.

16. Belair C, Baud J, Chabas S, et al. Helicobacter pylori interferes with an embryonic stem cell micro RNA cluster to block cell cycle progression. Silence 2011;2:7.

17. Salama N, Guillemin K, McDaniel TK, Sherlock G, Tompkins L, 
Falkow S. A whole-genome microarray reveals genetic diversity among Helicobacter pylori strains. Proc Natl Acad Sci U S A 2000;97:14668-14673.

18. Zhu Y, Jiang Q, Lou X, et al. MicroRNAs up-regulated by CagA of Helicobacter pylori induce intestinal metaplasia of gastric epithelial cells. PLoS One 2012;7:e35147.

19. Christman JK. 5-Azacytidine and 5-aza-2'-deoxycytidine as inhibitors of DNA methylation: mechanistic studies and their implications for cancer therapy. Oncogene 2002;21:5483-5495.

20. Yoshida M. Potent and specific inhibition of mammalian histone deacetylase both in vivo and in vitro by trichostatin A. Tanpakushitsu Kakusan Koso 2007;52(13 Suppl):1788-1789.

21. Tsai KW, Wu CW, Hu LY, et al. Epigenetic regulation of miR34b and miR-129 expression in gastric cancer. Int J Cancer 2011;129:2600-2610.

22. Fassi Fehri L, Koch M, Belogolova E, et al. Helicobacter pylori induces miR-155 in T cells in a cAMP-Foxp3-dependent manner. PLoS One 2010;5:e9500.

23. Xiao B, Liu Z, Li BS, et al. Induction of microRNA-155 during Helicobacter pylori infection and its negative regulatory role in the inflammatory response. J Infect Dis 2009;200:916-925.

24. Oertli M, Engler DB, Kohler E, Koch M, Meyer TF, Müller A. MicroRNA-155 is essential for the T cell-mediated control of Helicobacter pylori infection and for the induction of chronic gastritis and colitis. J Immunol 2011;187:3578-3586.

25. Liu Z, Xiao B, Tang B, et al. Up-regulated microRNA-146a negatively modulate Helicobacter pylori-induced inflammatory response in human gastric epithelial cells. Microbes Infect 2010;12:854-863.

26. Li N, Xu X, Xiao B, et al. H. pylori related proinflammatory cytokines contribute to the induction of miR-146a in human gastric epithelial cells. Mol Biol Rep 2012;39:4655-4661.

27. Gao C, Zhang Z, Liu W, Xiao S, Gu W, Lu H. Reduced microRNA-218 expression is associated with high nuclear factor kappa B activation in gastric cancer. Cancer 2010;116:41-49.

28. Feng Y, Wang L, Zeng J, et al. FoxM1 is overexpressed in Helicobacter pylori-induced gastric carcinogenesis and is negatively regulated by miR-370. Mol Cancer Res 2013;11:834-844.

29. Ishimoto $\mathrm{T}$, Izumi D, Watanabe $\mathrm{M}$, et al. Chronic inflammation with Helicobacter pylori infection is implicated in CD44 overexpression through miR-328 suppression in the gastric mucosa. J
Gastroenterol 2015;50:751-757.

30. Akao Y, Nakagawa Y, Naoe T. let-7 microRNA functions as a potential growth suppressor in human colon cancer cells. Biol Pharm Bull 2006;29:903-906.

31. Yanaihara N, Caplen N, Bowman E, et al. Unique microRNA molecular profiles in lung cancer diagnosis and prognosis. Cancer Cell 2006;9:189-198.

32. Higashi H, Tsutsumi R, Fujita A, et al. Biological activity of the Helicobacter pylori virulence factor CagA is determined by variation in the tyrosine phosphorylation sites. Proc Natl Acad Sci U S A 2002;99:14428-14433.

33. Azuma T, Yamazaki S, Yamakawa A, et al. Association between diversity in the Src homology 2 domain: containing tyrosine phosphatase binding site of Helicobacter pylori CagA protein and gastric atrophy and cancer. J Infect Dis 2004;189:820-827.

34. Brandt S, Kwok T, Hartig R, König W, Backert S. NF-kappaB activation and potentiation of proinflammatory responses by the Helicobacter pylori CagA protein. Proc Natl Acad Sci U S A 2005;102:9300-9305

35. Hayashi Y, Tsujii M, Wang J, et al. CagA mediates epigenetic regulation to attenuate let-7 expression in Helicobacter pylori-related carcinogenesis. Gut 2013;62:1536-1546.

36. Matsushima $\mathrm{K}$, Isomoto $\mathrm{H}$, Inoue $\mathrm{N}$, et al. MicroRNA signatures in Helicobacter pylori-infected gastric mucosa. Int J Cancer 2011;128:361-370.

37. Suzuki H, Yamamoto E, Nojima M, et al. Methylation-associated silencing of microRNA-34b/c in gastric cancer and its involvement in an epigenetic field defect. Carcinogenesis 2010;31:20662073.

38. Ando T, Yoshida T, Enomoto S, et al. DNA methylation of microRNA genes in gastric mucosae of gastric cancer patients: its possible involvement in the formation of epigenetic field defect. Int J Cancer 2009;124:2367-2374.

39. Shen J, Xiao Z, Wu WK, et al. Epigenetic silencing of miR-490$3 p$ reactivates the chromatin remodeler SMARCD1 to promote Helicobacter pylori-induced gastric carcinogenesis. Cancer Res 2015;75:754-765

40. Kiga K, Mimuro H, Suzuki M, et al. Epigenetic silencing of miR210 increases the proliferation of gastric epithelium during chronic Helicobacter pylori infection. Nat Commun 2014;5:4497. 